\title{
Structures of not-finitely graded Lie algebras related to generalized Virasoro algebras*
}

\author{
Qiufan Chen*, Jianzhi Han ${ }^{\dagger}$, Yucai Su* \\ *Department of Mathematics, Tongji University, Shanghai 200092, China \\ $\dagger$ Deparment of Mathematics, Sichuan University, Chengdu, Sichuan 610064, China
}

\begin{abstract}
In this paper, we study the structure theory of a class of not-finitely graded Lie algebras related to generalized Virasoro algebras. In particular, the derivation algebras, the automorphism groups and the second cohomology groups of these Lie algebras are determined.
\end{abstract}

Key words: not-finitely graded Lie algebras, generalized Virasoro algebras, derivations, automorphisms, 2-cocycles.

Mathematics Subject Classification (2010): 17B05, 17B40, 17B65, $17 \mathrm{~B} 68$.

\section{Introduction}

As it is well-known that the Virasoro algebra is an important object in mathematics and physics, whose theory has been widely used in literature (e.g., [2,3]). Various generalizations of the Virasoro algebra have been studied by several authors (e.g., [3-5,8-12]). In this paper, we consider the following Lie algebras $W(\Gamma)$ : Let $\Gamma$ be any nontrivial additive subgroup of $\mathbb{C}$, and $\mathbb{C}\left[\Gamma \times \mathbb{Z}_{+}\right]$the semigroup algebra of $\Gamma \times \mathbb{Z}_{+}$with basis $\left\{x^{\alpha, i}:=x^{\alpha} t^{i} \mid \alpha \in \Gamma, i \in \mathbb{Z}_{+}\right\}$ and product $x^{\alpha, i} x^{\beta, j}=x^{\alpha+\beta, i+j}$. Let $\partial_{x}, \partial_{t}$ be the derivations of $\mathbb{C}\left[\Gamma \times \mathbb{Z}_{+}\right]$defined by $\partial_{x}\left(x^{\alpha, i}\right)=\alpha x^{\alpha, i}, \partial_{t}\left(x^{\alpha, i}\right)=i x^{\alpha, i-1}$ for $\alpha \in \Gamma, i \in \mathbb{Z}_{+}$. Denote $\partial=\partial_{x}+t^{2} \partial_{t}$. Then the Lie algebra $W(\Gamma)$ is $\mathbb{C}\left[\Gamma \times \mathbb{Z}_{+}\right] \partial$ with basis $\left\{L_{\alpha, i}:=x^{\alpha, i} \partial \mid \alpha \in \Gamma, i \in \mathbb{Z}_{+}\right\}$and relation

$$
\left[L_{\alpha, i}, L_{\beta, j}\right]=(\beta-\alpha) L_{\alpha+\beta, i+j}+(j-i) L_{\alpha+\beta, i+j+1},
$$

for $\alpha, \beta \in \Gamma, i, j \in \mathbb{Z}_{+}$. One can realize the Lie algebra $W(\Gamma)$ as follows: Let $\mathcal{A}:=C_{[0,+\infty)}^{\infty}$ be the algebra consisting of smooth functions on variable $t$ in the interval $[0,+\infty)$, which becomes a Lie algebra under bracket $[f, g]=f g^{\prime}-f^{\prime} g$ for $f=f(t), g=g(t) \in \mathcal{A}$, where the prime stands for the derivative $\frac{d}{d t}$. Then $W(\Gamma)$ is the Lie subalgebra of $\mathcal{A}$ consisting of smooth functions $L_{\alpha, i}(t)=-\frac{e^{-\alpha t}}{(1+t)^{i}}, a \in \Gamma, i \in \mathbb{Z}_{+}$. Thus, $W(\Gamma)$ appears very naturally. One can observe that this algebra looks very similar to the Lie algebra $\mathcal{W}=W(0,1,0 ; \Gamma)$ of Witt type studied in [8], which has the same basis with bracket

$$
\left[L_{\alpha, i}, L_{\beta, j}\right]=(\alpha-\beta) L_{\alpha+\beta, i+j}+(j-i) L_{\alpha+\beta, i+j-1} .
$$

However, $W(\Gamma)$ has the following significant different features from that of $\mathcal{W}$ :

(i) The $\mathcal{W}$ is simple, but $W(\Gamma)$ has infinitely many ideals.

* Supported by NSF grant no. 11371278, 11001200 and 11101269 of China, the Fundamental Research Funds for the Central Universities, the grant no. 12XD1405000 of Shanghai Municipal Science and Technology Commission. ${ }^{\dagger}$ Supported by China Postdoctoral Science Foundation grant no. 2013M540709.

Corresponding author: J. Han (jzzhan@mail.ustc.edu.cn). 
(ii) The $\mathcal{W}$ has an ad-locally finite element $L_{0,0}$, but $W(\Gamma)$ does not have any nonzero ad-locally finite element.

(iii) The $\mathcal{W}$ has a finitely graded filtration in the sense that there exists a filtration $0 \subset$ $\mathcal{W}^{(0)} \subset \mathcal{W}^{(1)} \subset \cdots$, satisfying $\left[\mathcal{W}^{(i)}, \mathcal{W}^{(j)}\right] \subseteq \mathcal{W}^{(i+j)}$ for all $i, j \in \mathbb{Z}_{+}$and each $\mathcal{W}^{(i)}$ is finitely graded, i.e., there exists some abelian group $G$ which is independent of $i$ (one can simply choose $G=\Gamma$ in this case) such that

$$
\mathcal{W}^{(i)}=\bigoplus_{g \in G} \mathcal{W}_{g}^{(i)}, \quad\left[\mathcal{W}_{g}^{(i)}, \mathcal{W}_{h}^{(j)}\right] \subseteq \mathcal{W}_{g+h}^{(i+j)}, \quad \operatorname{dim} \mathcal{W}_{g}^{(i)}<\infty, \quad \text { and } \mathcal{W}_{0}^{(0)} \neq 0
$$

for all $g, h \in G, i, j \in \mathbb{Z}_{+}$(cf. (2.1)). On the other hand, $W(\Gamma)$ does not have any such finitely graded filtration for any abelian group. (See the first part of the proof of Theorem 2.1(2). One can also see this directly from the following: Fix $0 \neq x \in W_{0}^{(0)}$, take any $\beta \notin \operatorname{Supp} x \cap \Gamma$ (cf. (2.6) below) and assume that $L_{\beta, 1} \in \oplus_{g \in I} W_{g}^{(j)}$ for some $j \in \mathbb{Z}_{+}$and some finite subset $I$ of $G$. Then $\left\{\operatorname{ad}_{x}^{k} L_{\beta, 1} \mid k \in \mathbb{Z}_{+}\right\}$is an independent subset of $\oplus_{g \in I} W_{g}^{(j)}$, which forces some space $W_{g}^{(j)}$ to be infinite dimensional.)

The Lie algebra $W(\Gamma)$ has the unique universal central extension $\hat{W}(\Gamma)=W(\Gamma) \oplus \mathbb{C} C$ with one dimensional center $\mathbb{C} C$ and relation (cf. Theorem 5.1)

$$
\left[L_{\alpha, i}, L_{\beta, j}\right]=(\beta-\alpha) L_{\alpha+\beta, i+j}+(j-i) L_{\alpha+\beta, i+j+1}+\delta_{\alpha+\beta, 0} \delta_{i+j, 0} \frac{\alpha^{3}-\alpha}{12} C,
$$

for $\alpha, \beta \in \Gamma, i, j \in \mathbb{Z}_{+}$. Then $\hat{W}(\Gamma)$ contains the (generalized) Virasoro algebra

$$
\operatorname{Vir}(\Gamma)=\operatorname{span}\left\{L_{\alpha, 0}, C \mid \alpha \in \Gamma\right\}
$$

We refer to $\hat{W}(\Gamma)$ as a not-finitely graded (generalized) Virasoro algebra (see Theorem 2.1(1)). Not-finitely graded Lie algebras are important objects in Lie theory, whose structure and representation theories are subjects of studies with more challenge than that of finitely graded Lie algebras. The Lie algebra $\hat{W}(\Gamma)$ is interesting to us in another aspect that it is also closely related to Lie algebras of Block type (e.g., [6,7]), and it contains some interesting properties as stated in Theorem 2.1. Furthermore it also contains many interesting (finitely) $\Gamma$-graded subquotient algebras $\tilde{W}^{m, n}$ for $n \geq m \geq 0$, where

$$
\tilde{W}^{m, n}=W^{m} / W^{n+1}, \quad W^{m}=\operatorname{span}\left\{L_{\alpha, i} \mid \alpha \in \Gamma, i \geq m\right\} \oplus \delta_{m, 0} \mathbb{C} C .
$$

For instance, $\tilde{W}^{0,0}$ is the (generalized) Virasoro algebra $\operatorname{Vir}(\Gamma)$ (thus $\operatorname{Vir}(\Gamma)$ is both a subalgebra and a quotient algebra of $\hat{W}(\Gamma)$ ), $\tilde{W}^{0,1}$ is the well-known $W$-algebra $W(2,2)$ (up to different central extensions, see, e.g., [13]). When one studies the representation theory, it 
is well-known that the category of modules of a quotient Lie algebra $\tilde{W}^{0, n}$ is a full subcategory of the category of $\hat{W}(\Gamma)$-modules. Thus a study of $W$-modules will lead to a study of $\tilde{W}^{0, n}$-modules for all $n>0$.

In this paper we shall mainly study the structure theory (namely, derivations, automorphisms, 2-cocycles), in this case the central element $C$ does not play a crucial role, thus we shall only consider $W(\Gamma)$ instead of $\hat{W}(\Gamma)$, and we simply denote $W:=W(\Gamma)$. We shall study the representation theory of $\hat{W}(\Gamma)$ in a sequel paper. The Lie algebra $W$ is $\Gamma$-graded

$$
W=\bigoplus_{\alpha \in \Gamma} W_{\alpha}, \quad W_{\alpha}=\operatorname{span}\left\{L_{\alpha, i} \mid i \in \mathbb{Z}_{+}\right\} \text {for } \alpha \in \Gamma
$$

However, it is not finitely graded. Nevertheless, due to the fact that $\Gamma$ may not be finitely generated (as a group), and so $W$ may not be finitely generated as a Lie algebra, the classical techniques (such as those in [1]) cannot be directly applied to our situation here. One must employ some new techniques in order to tackle problems associated with not-finitely graded and not-finitely generated Lie algebras (this is also one of our motivations to present our results here). For instance, one of our strategies used in the present paper is to embed $W$ into its completed algebra $\bar{W}$ (see (3.3)) so that the determination of derivations can be done much more efficiently than that in some classical methods in the literature (e.g., [11]). The main results of the present paper are summarized in Theorems 2.1, 3.1, 4.1 and 5.1.

Throughout the paper, we denote by $\mathbb{C}, \mathbb{C}^{*}, \mathbb{Z}, \mathbb{Z}_{+}, \Gamma^{*}$ the sets of complex numbers, nonzero complex numbers, integers, nonnegative integers, nonzero elements of $\Gamma$ respectively.

\section{Some properties of $W(\Gamma)$}

We first study some properties of the Lie algebra $W:=W(\Gamma)$, which will be summarized in Theorem 2.1. First we recall some concepts. A Lie algebra $\mathcal{L}$ is finitely graded if there exists an abelian group $G$ such that $\mathcal{L}=\oplus_{a \in G} \mathcal{L}_{[a]}$ is $G$-graded satisfying

$$
\left[\mathcal{L}_{[a]}, \mathcal{L}_{[b]}\right] \subset \mathcal{L}_{[a+b]} \text { and } \operatorname{dim} \mathcal{L}_{[a]}<\infty \text { for } a, b \in G
$$

An element $x \in \mathcal{L}$ is ad-locally finite if for any $y \in \mathcal{L}$ the $\operatorname{subspace} \operatorname{span}\left\{\operatorname{ad}_{x}^{i}(y) \mid i \in \mathbb{Z}_{+}\right\}$is finite-dimensional, where $\operatorname{ad}_{x}: y \mapsto[x, y](y \in \mathcal{L})$ is the adjoint operator of $x$.

By (1.4) (we regard $C$ as zero), we have the following filtration of ideals,

$$
W=W^{0} \supset W^{1} \supset W^{2} \supset \cdots .
$$

Theorem 2.1. (1) The Lie algebra $W$ does not contain any nonzero ad-locally finite element.

(2) The Lie algebra $W$ is not finitely graded. 
(3) The $W^{n}$ with $n \in \mathbb{Z}_{+}$are all of the nonzero ideals of $W$ (thus $W^{1}$ is the unique maximal ideal of $W$ ). Furthermore,

$$
\left.\left.W^{n}=\operatorname{ad}_{W^{1}}^{m}\left(W^{n-m}\right)=[\overbrace{W^{1},\left[W^{1}, \ldots,\left[W^{1}\right.\right.}^{m}, W^{n-m}] \ldots\right]\right] \text { for any } 0 \leq m \leq n .
$$

Proof. (1) Suppose $0 \neq x=\sum_{\alpha \in \Gamma, i \in \mathbb{Z}_{+}} a_{\alpha, i} L_{\alpha, i}$ (finite sum) for some $a_{\alpha, i} \in \mathbb{C}$ is an ad-locally finite element. Choose a total order " $\prec$ " on $\Gamma$ compatible with its group structure, and define the total order on $\Gamma \times \mathbb{Z}_{+}$by

$$
(\alpha, i) \prec(\beta, j) \Longleftrightarrow \quad \Longleftrightarrow \prec \beta \text { or } \alpha=\beta, i<j
$$

for $(\alpha, i),(\beta, j) \in \Gamma \times \mathbb{Z}_{+}$. Let $\left(\alpha_{0}, i_{0}\right)=\max \left\{(\alpha, i) \mid a_{\alpha, i} \neq 0\right\}$. Take $y=L_{0, j}$ with $j=\delta_{i_{0}, 0}$. Then for different $k$, the element $\operatorname{ad}_{x}^{k}(y)$ has the different highest term

$$
\prod_{p=0}^{k-1}\left(j-i_{0}+p\left(i_{0}+1\right)\right) a_{\alpha_{0}, i_{0}}^{k} L_{k \alpha_{0}, j+k\left(i_{0}+1\right)} \neq 0
$$

i.e., $\operatorname{ad}_{x}^{k}(y)$ for $k=0,1,2, \ldots$, are linearly independent, which is a contradiction with

$$
\operatorname{dim} \operatorname{span}\left\{\operatorname{ad}_{x}^{i}(y) \mid i \in \mathbb{Z}_{+}\right\}<\infty
$$

(2) Suppose there exists some abelian group $G$ such that $W=\oplus_{g \in G} W_{[g]}$ is $G$-graded satisfying (2.1). Suppose $W_{[0]} \neq 0$. Take any nonzero $x \in W_{[0]}$ and $\beta \in \Gamma$ such that $\beta \notin \operatorname{Supp} x$ (cf. (2.6) below). Assume that $L_{\beta, 1} \in \oplus_{g \in I} W_{[g]}$ for some finite subset $I$ of $G$. Then it follows from the proof of (1) that $\left\{\operatorname{ad}_{x}^{k} L_{\beta, 1} \mid k \in \mathbb{Z}_{+}\right\}$is an independent subset of $\oplus_{g \in I} W_{[g]}$. In particular, $\sum_{g \in I} \operatorname{dim} W_{[g]}=\infty$. Since $I$ is finite, there exists some $g_{0} \in I$ such that $\operatorname{dim} W_{\left[g_{0}\right]}=\infty$, contradicting our assumption that $W_{[g]}$ is finite dimensional for all $g \in G$.

So in the following we assume that $W_{[0]}=0$. Choose a total order " $\prec$ " on $G$ compatible with its group structure. Write $L_{0,0}=\sum_{i=1}^{n} x_{g_{i}}$ with $g_{n} \succ g_{n-1} \succ \cdots \succ g_{1}$ for some $n \geq 1$, where $0 \neq x_{g_{i}} \in W_{\left[g_{i}\right]}$. Since

$$
\left[L_{0,0}, L_{\alpha, 0}\right]=\alpha L_{\alpha, 0}
$$

that is, $L_{0,0}$ preserves each one dimensional space $\mathbb{C} L_{\alpha, 0}(\alpha \in \Gamma), L_{0,0}$ can not lie in the positive part $\oplus_{0 \prec g \in G} W_{[g]}$ or the negative part $\oplus_{0 \succ g \in G} W_{[g]}$ of $W$. So $g_{n} \succ 0$ and $g_{1} \prec 0$.

By comparing the maximal homogenous components (with respect to the $G$-gradation of $W$ ) of both sides of (2.5) we see that the maximal homogenous component of $L_{\alpha, 0}$ lies in $W_{\left[g_{n}\right]}$ for all $\alpha \in \Gamma$. So there exists $\lambda_{\alpha} \in \mathbb{C}^{*}$ such that $L_{\alpha, 0}=\lambda_{\alpha} L_{0,0}+\sum_{g \prec g_{n}} y_{g}^{\alpha}$, where $y_{g}^{\alpha} \in W_{[g]}$. In fact, substituting $L_{\alpha, 0}=\lambda_{\alpha} L_{0,0}+\sum_{g \prec g_{n}} y_{g}^{\alpha}$ in the left hand side of (2.5) and then proceeding the analysis above give $L_{\alpha, 0}=\lambda_{\alpha} L_{0,0}+\sum_{g \prec 0} y_{g}^{\alpha}$. Furthermore, comparing 
the minimal homogenous component shows that $L_{\alpha, 0}=\lambda_{\alpha} L_{0,0}+y_{g_{1}}^{\alpha}$ for some $y_{g_{1}}^{\alpha} \in W_{\left[g_{1}\right]}$. In this case, we have

$$
\infty=\operatorname{dim}\left(\operatorname{span}\left\{L_{\alpha, 0}-\lambda_{\alpha} L_{0,0} \mid \alpha \in \Gamma\right\}\right) \leq \operatorname{dim} W_{\left[g_{1}\right]}
$$

contradicting the finite dimension of $W_{\left[g_{1}\right]}$.

(3) Suppose $A$ is a nonzero ideal, and $0 \neq x=\sum_{\alpha \in \Gamma} x_{\alpha} \in A$ (finite sum) such that each homogeneous component $x_{\alpha}=\sum_{i \in \mathbb{Z}_{+}} a_{\alpha, i} L_{\alpha, i}$ (finite sum) for some $a_{\alpha, i} \in \mathbb{C}$. Let

$$
\operatorname{Supp} x=\left\{\alpha \in \Gamma \mid x_{\alpha} \neq 0\right\} \quad(\text { called the support of } x) \text {. }
$$

We call the size $|\operatorname{Supp} x|$ is the depth of $x$. We want to prove the following claim by induction on the depth of $x$.

Claim 1. There exists some basis element $L_{\beta_{0}, j_{0}} \in A$.

First suppose $|\operatorname{Supp} x|=1$, i.e., $\operatorname{Supp} x=\{\alpha\}$ for some $\alpha \in \Gamma$. Thus $x$ is a homogeneous element and we can simply write $x=x_{\alpha}=\sum_{k=i}^{j} a_{k} L_{\alpha, k}$ for some $i \leq j$ such that $a_{i}, a_{j} \neq 0$. We say $a_{i} L_{\alpha, i}, a_{j} L_{\alpha, j}$ are respectively the first, last term of $x_{\alpha}$, and $i, j$ the first, last index of $x_{\alpha}$. We denote

$$
\ell(x)=j-i+1 \text { (called the length of the homogeneous element } x \text { ). }
$$

If $\ell(x)=1$, we have the claim. Thus suppose $\ell(x)>1$. Take

$$
y=\left[L_{\alpha, j}, x\right]=\sum_{k=i}^{j-1} a_{k}(k-j) L_{2 \alpha, j+k+1} \in A .
$$

We see that $y \neq 0$ is a homogeneous element such that $\ell(y)<\ell(x)$. The claim is obtained by induction on the length $\ell(x)$.

Now suppose $|\operatorname{Supp} x|>1$. Take a nonzero homogeneous component $x_{\alpha} \neq 0$ and set

$$
y:=\left[x_{\alpha}, x\right]=\sum_{\beta \in \operatorname{Supp} x, \beta \neq \alpha}\left[x_{\alpha}, x_{\beta}\right] \in A .
$$

One can easily see from (1.1) that the first term in $\left[x_{\alpha}, x_{\beta}\right]$ is nonzero if $\beta \neq \alpha$, thus $y \neq 0$ and $\operatorname{Supp} y=\{\alpha+\beta \mid \beta \in(\operatorname{Supp} x) \backslash\{\alpha\}\}$ has depth $|\operatorname{Supp} x|-1$. The claim is proved by induction on the depth of $x$.

Now suppose $j_{0}$ is smallest such that Claim 1 holds for some $\beta_{0} \in \Gamma$. Fix any $\gamma \in \Gamma^{*}$. For any $\beta \in \Gamma$, we define the operator

$$
\theta_{\beta}:=\operatorname{ad}_{L_{\beta-\gamma, 0}} \operatorname{ad}_{L_{\gamma, 0}}-2 \operatorname{ad}_{L_{\beta, 0}} \operatorname{ad}_{L_{0,0}}+\operatorname{ad}_{L_{\beta+\gamma, 0}} \operatorname{ad}_{L_{-\gamma, 0}} .
$$

Since $A$ is an ideal, applying $\theta_{\beta}$ to $L_{\beta_{0}, j_{0}}$, we see that $-4 \gamma^{2} L_{\beta+\beta_{0}, j_{0}}=\theta_{\beta}\left(L_{\beta_{0}, j_{0}}\right) \in A$. From this one can deduce that $L_{\beta, j} \in A$ for all $(\beta, j) \in \Gamma \times \mathbb{Z}_{+}$with $j \geq j_{0}$. Hence, $A \supset W^{j_{0}}$. If $j_{0}=0$, then $A=W^{0}=W$. So we assume $j_{0} \geq 1$ in the following. 
Suppose $A \nsubseteq W^{j_{0}}$. Consider the nonzero quotient space $A / W^{j_{0}}$ and denote by $\bar{x}$ the image of $x$ in $A / W^{j_{0}}$ for any $x \in A$. Then we can take $0 \neq \bar{x}=\sum_{\alpha \in I, 0 \leq i \leq j_{0}-1} c_{\alpha, i} \bar{L}_{\alpha, i}$, where $I$ is a finite subset of $\Gamma$. Set $i_{0}=\min \left\{i \mid c_{\alpha, i} \neq 0, \alpha \in I\right\}$. Fix any $\beta \in \Gamma-I$, then one can see that the image of $y_{\beta}:=\left[L_{\beta, j_{0}-i_{0}-1}, x\right]$ is nonzero in $W^{j_{0}-1} / W^{j_{0}} \bigcap A / W^{j_{0}}$. Observe that $\bar{L}_{0,0}$ acts semisimply on $W^{j_{0}-1} / W^{j_{0}}$. Repeatedly applying the action of $\bar{L}_{0,0}$ on $\bar{y}_{\beta}$ will yield that $L_{\alpha+\beta, j_{0}-1} \in A$ for $\alpha \in I$ such that $c_{\alpha, i_{0}} \neq 0$, a contradiction with the minimality of $j_{0}$.

\section{Derivation algebra}

Recall that a linear map $D: W \rightarrow W$ is a derivation of $W$ if $D([x, y])=[D(x), y]+[x, D(y)]$ for any $x, y \in W$. For any $z \in W$, the adjoint operator $\operatorname{ad}_{z}: W \rightarrow W$ is a derivation, called an inner derivation. Denote by Der $W$ and ad $W$ the vector spaces of all derivations and inner derivations respectively. Then the first cohomology group $H^{1}(W, W) \cong \operatorname{Der} W / \operatorname{ad} W$.

Let $\operatorname{Hom}_{\mathbb{Z}}(\Gamma, \mathbb{C})$ denote the space of group homomorphisms from $\Gamma$ to (the additive group) $\mathbb{C}$ (for each $\phi \in \operatorname{Hom}_{\mathbb{Z}}(\Gamma, \mathbb{C})$, the scalar multiplication $\phi$ by $c \in \mathbb{C}$ is defined by $(c \phi)(\gamma)=c \phi(\gamma)$, thus $\operatorname{Hom}_{\mathbb{Z}}(\Gamma, \mathbb{C})$ is a vector space). For each $\phi \in \operatorname{Hom}_{\mathbb{Z}}(\Gamma, \mathbb{C})$, we can define a derivation $D_{\phi}$ as follows,

$$
D_{\phi}\left(L_{\alpha, i}\right)=\phi(\alpha) L_{\alpha, i} \quad \text { for } \quad \alpha \in \Gamma, i \in \mathbb{Z}_{+} .
$$

We still use $\operatorname{Hom}_{\mathbb{Z}}(\Gamma, \mathbb{C})$ to denote the corresponding subspace of Der $W$. In particular, since $\phi_{0}: \alpha \mapsto \alpha$ is in $\operatorname{Hom}_{\mathbb{Z}}(\Gamma, \mathbb{C})$, we have the derivation

$$
D_{0}=D_{\phi_{0}}: L_{\alpha, i} \mapsto \alpha L_{\alpha, i} \text { for } \alpha \in \Gamma, i \in \mathbb{Z}_{+} .
$$

Theorem 3.1. We have $\operatorname{Der} W=\operatorname{ad} W \oplus \operatorname{Hom}_{\mathbb{Z}}(\Gamma, \mathbb{C})$. In particular, if $\Gamma=\mathbb{Z}$, we have Der $W(\mathbb{Z})=\operatorname{ad} W(\mathbb{Z}) \oplus \mathbb{C} D_{0}$.

Proof. First by noting that every element in $\operatorname{Hom}_{\mathbb{Z}}(\Gamma, \mathbb{C})$ acts locally-finitely on $W$, and by Theorem 2.1(2), we can easily prove that the sum in the theorem is direct. Since $\operatorname{Hom}_{\mathbb{Z}}(\mathbb{Z}, \mathbb{C})=\mathbb{C} \phi_{0}$, we have the second statement.

Now take $\bar{W}$ to be the space whose elements are finite sums of homogeneous elements $x_{\alpha}$ for $\alpha \in \Gamma$ (i.e., each element of $\bar{W}$ has a finite support, cf. (2.6)), and each $x_{\alpha}$ has the form $x_{\alpha}=\sum_{i \in \mathbb{Z}_{+}} a_{\alpha, i} L_{\alpha, i}$ (which can be an infinite sum, i.e., each homogeneous element can have infinite length, cf. (2.7)). Then $\bar{W}$ becomes a Lie algebra with bracket defined by (cf. (1.1))

$$
\left[\sum_{\alpha, i} a_{\alpha, i} L_{\alpha, i}, \sum_{\beta, j} b_{\beta, j} L_{\beta, j}\right]=\sum_{\alpha, \beta, i, j} a_{\alpha, i} b_{\beta, j}\left((\beta-\alpha) L_{\alpha+\beta, i+j}+(j-i) L_{\alpha+\beta, i+j+1}\right) .
$$

Clearly $W \subset \bar{W}$. We call $\bar{W}$ the completion of $W$. Let $D \in \operatorname{Der} W$. Applying $D$ to $\left[L_{0,0}, L_{\alpha, i}\right]=\alpha L_{\alpha, i}+i L_{\alpha, i+1}$ one can see that

$$
\operatorname{Supp}\left(D\left(L_{\alpha, i+1}\right)\right) \subseteq \operatorname{Supp}\left(\left(D L_{\alpha, i}\right)\right) \cup\left(\operatorname{Supp}\left(D\left(L_{0,0}\right)+\alpha\right)\right.
$$


for any $i \geq 1$. Inductively, we have

$$
\operatorname{Supp}\left(D\left(L_{\alpha, i+1}\right)\right) \subseteq \operatorname{Supp}\left(D\left(L_{\alpha, 1}\right)\right) \cup\left(\operatorname{Supp}\left(D\left(L_{0,0}\right)+\alpha\right)\right.
$$

for any $i \geq 1$. So $\sum_{i \in \mathbb{Z}_{+}} a_{i} D\left(L_{\alpha, i}\right) \in \bar{W}$. Thus if we define

$$
\bar{D}\left(\sum_{\alpha, i} a_{\alpha, i} L_{\alpha, i}\right)=\sum_{\alpha, i} a_{\alpha, i} D\left(L_{\alpha, i}\right)
$$

for any $\sum_{\alpha, i} a_{\alpha, i} L_{\alpha, i} \in \bar{W}$, then $\bar{D} \in \operatorname{End} \bar{W}$; Moreover, $\bar{D} \in \operatorname{Der} \bar{W}$. We shall prove the result in two steps:

(i) First by replacing $\bar{D}$ by $\bar{D}-\operatorname{ad}_{y}-D_{\phi}$ (here we simply denote $\bar{D}_{\phi}$ by $D_{\phi}$ ) for some $y \in \bar{W}, \phi \in \operatorname{Hom}_{\mathbb{Z}}(\Gamma, \mathbb{C})$, we want to prove $\left.\bar{D}\right|_{W}=0$.

(ii) Then we want to prove that in fact $y \in W$.

Suppose $\bar{D}\left(L_{0,0}\right)=\sum_{\alpha, i} a_{\alpha, i} L_{\alpha, i} \in W$ for some $a_{\alpha, i} \in \mathbb{C}$. For any $\alpha \in \Gamma$, we define $b_{\alpha, j} \in \mathbb{C}$ inductively on $j \geq 0$ by regarding $b_{\alpha,-1}$ as zero and

$$
b_{\alpha, j}= \begin{cases}\frac{1}{\alpha}\left(-a_{\alpha, j}-b_{\alpha, j-1}(j-1)\right) & \text { if } \alpha \neq 0, \\ -\frac{1}{j} a_{0, j+1} & \text { if } \alpha=0, j>0 \\ 0 & \text { if } \alpha=j=0 .\end{cases}
$$

Take $y=\sum_{\alpha, j} b_{\alpha, j} L_{\alpha, j}$. Note that $y \in \bar{W}$ (since the depth of $y$ is $|\operatorname{Supp} y| \leq|\operatorname{Supp} x|$, cf. (2.6)), and (3.4) gives

$$
\begin{aligned}
\bar{D}\left(L_{0,0}\right)-\operatorname{ad}_{y}\left(L_{0,0}\right) & =\sum_{\alpha, j} a_{\alpha, j} L_{\alpha, j}-\sum_{\alpha, j}\left(-b_{\alpha, j} \alpha-b_{\alpha, j-1}(j-1)\right) L_{\alpha, j} \\
& =a_{0,0} L_{0,0}+a_{0,1} L_{0,1} .
\end{aligned}
$$

This proves the following claim.

Claim 1. By replacing $\bar{D}$ by $\bar{D}-\operatorname{ad}_{y}$ for some $y \in \bar{W}$, we can suppose

$$
\bar{D}\left(L_{0,0}\right)=a_{0} L_{0,0}+a_{1} L_{0,1} \text { for some } a_{0}, a_{1} \in \mathbb{C}
$$

For any $\alpha \in \Gamma$, assume $\bar{D}\left(L_{\alpha, 0}\right)=\sum_{\beta, j} a_{\beta, j}^{\alpha} L_{\beta, j}$ for some $a_{\beta, j}^{\alpha} \in \mathbb{C}$. Applying $\bar{D}$ to $\left[L_{0,0}, L_{\alpha, 0}\right]=\alpha L_{\alpha, 0}$ and comparing the coefficients of $L_{\beta, k}$, by induction on $k$ we obtain $a_{\beta, k}^{\alpha}=0$ for all $k \geq 0$ if $\beta \neq \alpha$ and $a_{\alpha, k}^{\alpha}=0$ for all $k \geq 1$, and we also obtain $a_{0}=a_{1}=0$. Thus we can assume $\bar{D}\left(L_{\alpha, 0}\right)=b_{\alpha} L_{\alpha, 0}$ for some $b_{\alpha} \in \mathbb{C}$ such that by $(3.5)$,

$$
b_{0}=0
$$


Applying $\bar{D}$ to $\left[L_{\alpha, 0}, L_{\gamma, 0}\right]=(\gamma-\alpha) L_{\alpha+\gamma, 0}$, we obtain that the following holds for $\alpha \neq \gamma$,

$$
b_{\alpha+\gamma}=b_{\alpha}+b_{\gamma} .
$$

In particular, $b_{-\alpha}=-b_{\alpha}$ for all $\alpha \in \Gamma$. If $\alpha=\gamma$, we have $b_{2 \alpha}=b_{(\alpha+\eta)+(\alpha-\eta)}=b_{\alpha+\eta}+b_{\alpha+(-\eta)}=$ $b_{\alpha}+b_{\eta}+b_{\alpha}+b_{-\eta}=2 b_{\alpha}$ for any $\eta \in \Gamma \backslash\{ \pm \alpha, 0\}$. Thus (3.7) holds for all $\alpha, \gamma \in \Gamma$, which shows that the map $\phi: \alpha \mapsto b_{\alpha}$ is an element in $\operatorname{Hom}_{\mathbb{Z}}(\Gamma, \mathbb{C})$. If we replace $\bar{D}$ by $\bar{D}-D_{\phi}$ (cf. (3.1)) (note that this replacement does not affect (3.5)), we can suppose $b_{\alpha}=0$ for all $\alpha \in \Gamma$. This proves the following claim.

Claim 2. Replacing $\bar{D}$ by $\bar{D}-D_{\phi}$ for some $D_{\phi} \in \operatorname{Hom}_{\mathbb{Z}}(\Gamma, \mathbb{C})$, we can suppose $\bar{D}\left(L_{\alpha, 0}\right)=0$ for all $\alpha \in \Gamma$.

For any $D \in$ Der $W$ and $\triangle \in \Gamma$, define the homogeneous operator $\bar{D}_{\triangle}$ of degree $\triangle$ in the following way

$$
\bar{D}_{\triangle}\left(\sum_{\alpha} u_{\alpha}\right)=\sum_{\alpha} \pi_{\alpha+\triangle} \bar{D} u_{\alpha}
$$

where $u_{\alpha} \in \bar{W}_{\alpha}$ and $\pi_{\alpha}: \bar{W} \rightarrow \bar{W}_{\alpha}$ is the natural projection. Then $\bar{D}=\sum_{\triangle \in \Gamma} \bar{D}_{\triangle}$ and $\bar{D}_{\triangle} \in \operatorname{Der} \bar{W}$. For any $\alpha \in \Gamma$, suppose

$$
\bar{D}_{\triangle} L_{\alpha, 1}=\sum_{j} c_{\alpha, j}^{\Delta} L_{\alpha+\triangle, j}\left(c_{\alpha, j}^{\triangle} \in \mathbb{C}\right) .
$$

Applying $\bar{D}_{\triangle}$ to $\left[L_{\alpha, 0}, L_{\beta, 1}\right]-\left[L_{0,0}, L_{\alpha+\beta, 1}\right]=-2 \alpha L_{\alpha+\beta, 1}$, one has

$$
(\beta+\triangle-\alpha)\left(c_{\alpha+\beta, j}^{\Delta}-c_{\beta, j}^{\Delta}\right)=(1-j)\left(c_{\alpha+\beta, j-1}^{\Delta}-c_{\beta, j-1}^{\Delta}\right)\left(\text { we take } c_{\alpha,-1}^{\Delta}=0\right) .
$$

It follows that $c_{\alpha+\beta, j}^{\Delta}=c_{\beta, j}^{\Delta}$ for any $j \geq 0$ whenever $\beta+\triangle-\alpha \neq 0$ and $c_{2 \beta+\triangle, j}^{\Delta}=c_{\beta, j}^{\Delta}$ for any $j \geq 1$. In particular, take $\beta=0$ and $\alpha+\beta=0$ respectively, then one can see that $c_{\gamma, j}^{\Delta}=c_{0, j}^{\Delta}$ for any $\gamma \in \Gamma$ and $j \geq 0$. This means the coefficient $c_{\gamma, j}^{\Delta}$ is independent of the choice of $\gamma$. So in the following, for convenience, we can drop off the symbol $\gamma$ and just write $c_{j}^{\triangle}$ instead of $c_{\gamma, j}^{\Delta}$. Note that $\left[L_{\alpha, 1}, L_{\beta, 1}\right]+(\alpha-\beta)\left[L_{0,0}, L_{\alpha+\beta, 1}\right]=\left(\alpha^{2}-\beta^{2}\right) L_{\alpha+\beta, 1}$. By applying $\bar{D}_{\triangle}$ to this equation and comparing the coefficients of $L_{\alpha+\beta+\triangle, j}$, we obtain $\triangle c_{j}^{\Delta}=(3-j) c_{j-1}^{\Delta}$, which gives rise to $c_{j}^{\Delta}=0$ for any $\triangle \neq 0$ and $j \geq 0$ and $c_{k}^{0}=0$ whenever $k \neq 2$. Hence the equation (3.8) can be simply written as

$$
\bar{D}_{\triangle} L_{\alpha, 1}=c \delta_{\triangle, 0} L_{\alpha, 2}(c \in \mathbb{C}) .
$$

Applying $\bar{D}_{0}$ to $\left[L_{\alpha, 1}, L_{\beta, 1}\right]=(\beta-\alpha) L_{\alpha+\beta, 2}$, one can immediately see that $\bar{D}_{0} L_{\alpha, 2}=c L_{\alpha, 3}$. By applying $\bar{D}_{0}$ to $\left[L_{0,0}, L_{\alpha, 1}\right]-\alpha L_{\alpha, 1}=L_{\alpha, 2}$ we obtain $\bar{D}_{0} L_{\alpha, 3}=3 c L_{\alpha, 4}$. By induction, 
we can obtain that $\bar{D}_{0} L_{\alpha, i}=c i L_{\alpha, i+1}$, that is to say $\bar{D}_{0}=c\left(\operatorname{ad}_{L_{0,0}}-D_{0}\right)$. Replacing $y$ by $y-c L_{0,0}$ and $\phi$ by $\phi^{\prime}:=\phi+c \phi_{0}$ respectively (which does not affect $\bar{D}\left(L_{\alpha, 0}\right)=0$ ), we get $\bar{D} L_{\alpha, 1}=0$. So far, we have proved that $\bar{D} L_{\alpha, 0}=\bar{D} L_{\alpha, 1}=0$ for all $\alpha \in \Gamma$. Since $W$ is generated by $\left\{L_{\alpha, 0}, L_{\alpha, 1} \mid \alpha \in \Gamma\right\}$, we obtain $\left.\bar{D}\right|_{W}=0$, and the first step is completed.

The first step in fact shows that $D=\left.\bar{D}\right|_{W}=\left.\left(\operatorname{ad}_{y}+D_{\phi}\right)\right|_{W}$. If $y \notin W$, then some homogeneous component, say, $y_{\alpha}=\sum_{i \geq 0} a_{i} L_{\alpha, i}$ (for some $a_{i} \in \mathbb{C}$ ), of $y$ is not in $W$. Choose $\beta \in \Gamma^{*}$, and set $u=D\left(L_{\beta, 0}\right), w=D\left(L_{2 \beta, 0}\right) \in W$. Then the homogeneous components

$$
\begin{aligned}
& u_{\alpha+\beta}=-\sum_{i \geq 0}\left(a_{i}(\alpha-\beta)+(i-1) a_{i-1}\right) L_{\alpha+\beta, i}+\delta_{\alpha, 0} D_{\phi^{\prime}} L_{\beta, 0}\left(\text { we take } a_{-1}=0\right), \\
& w_{\alpha+2 \beta}=-\sum_{i \geq 0}\left(a_{i}(\alpha-2 \beta)+(i-1) a_{i-1}\right) L_{\alpha+2 \beta, i}+\delta_{\alpha, 0} D_{\phi^{\prime}} L_{2 \beta, 0},
\end{aligned}
$$

are in $W$. Thus there exists some $N>0$ such that $a_{i}(\alpha-\beta)+(i-1) a_{i-1}=a_{i}(\alpha-2 \beta)+$ $(i-1) a_{i-1}=0$ for $i>N$. In particular $a_{i}=0$ for $i>N$, a contradiction with the fact that $y_{\alpha} \notin W$. This completes the proof of the second step, thus the theorem.

\section{Automorphism group}

Denote by Aut $W$ the automorphism group of $W$. Let $\chi(\Gamma)$ be the set of characters of $\Gamma$, i.e., the set of group homomorphisms $\tau: \Gamma \rightarrow \mathbb{C}^{*}$. Set $\Gamma^{\mathbb{C}^{*}}=\left\{c \in \mathbb{C}^{*} \mid c \Gamma=\Gamma\right\}$. We define a group structure on $\chi(\Gamma) \times \Gamma^{\mathbb{C}^{*}}$ by

$$
\left(\tau_{1}, c_{1}\right) \cdot\left(\tau_{2}, c_{2}\right)=\left(\tau, c_{1} c_{2}\right), \text { where } \tau: \alpha \mapsto \tau_{1}\left(c_{2} \alpha\right) \tau_{2}(\alpha) \text { for } \alpha \in \Gamma \text {. }
$$

It turns out that the group $\chi(\Gamma) \times \Gamma^{\mathbb{C}^{*}}$ is just the semidirect product $\chi(\Gamma) \rtimes \Gamma^{\mathbb{C}^{*}}$ under the action given by $(c \tau)(\alpha)=\tau(c \alpha)$ for all $c \in \Gamma^{*}, \tau \in \chi(\Gamma), \alpha \in \Gamma$. We define a group homomorphism $\phi:(\tau, c) \mapsto \phi_{\tau, c}$ from $\chi(\Gamma) \times \Gamma^{\mathbb{C}^{*}}$ to Aut $W$ such that $\phi_{\tau, c}$ is the automorphism of $W$ defined by

$$
\phi_{\tau, c}: L_{\alpha, i} \mapsto \tau(\alpha) c^{-i-1} L_{c \alpha, i} \text { for } \alpha \in \Gamma, i \in \mathbb{Z}_{+} .
$$

One can easily verify that $\phi_{\tau, c}$ is indeed an automorphism of $W$.

Theorem 4.1. We have $\phi$ : Aut $W \cong \chi(\Gamma) \rtimes \Gamma^{\mathbb{C}^{*}}$.

Proof. Let $\sigma \in$ Aut $W$. Since $W^{1}$ is the unique maximal ideal of $W$, we have $\sigma\left(W^{1}\right)=W^{1}$. Then by (2.3), we obtain $\sigma\left(W^{n}\right)=W^{n}$ for all $n \in \mathbb{Z}_{+}$. In particular, $\sigma$ induces an automorphism $\bar{\sigma}$ of $\tilde{W}^{0,0} \cong \operatorname{Vir}(\Gamma)\left(\operatorname{cf}\right.$. (1.3) and (1.4)). Therefore $\sigma\left(L_{\alpha, 0}\right) \equiv \tau(\alpha) c^{-1} L_{c \alpha, 0}\left(\bmod W^{1}\right)$ for $\alpha \in \Gamma$ and some $\tau \in \chi(\Gamma), c \in \Gamma^{\mathbb{C}^{*}}$ (e.g., [11]), this result can also be proved directly by noting that $\mathbb{C}^{*} L_{0,0}$ is the set of nonzero ad-locally finite elements in $\operatorname{Vir}(\Gamma)$ ). By replacing $\sigma$ by $\sigma \phi_{\tau, c}^{-1}$, we can suppose $\tau=1, c=1$. Thus

$$
\sigma\left(L_{\alpha, 0}\right)-L_{\alpha, 0} \in W^{1}
$$


Claim 1. We have $\sigma\left(L_{0,0}\right)=L_{0,0}$.

First suppose that there exists some $\alpha \in \Gamma^{*}$ such that the homogeneous component $\sigma\left(L_{0,0}\right)_{\alpha}$ of $\sigma\left(L_{0,0}\right)$ is nonzero (we always use the same symbol with subscript $\alpha$ to denote its homogeneous component of degree $\alpha$ ). Recall from (2.6) that the support of $\sigma\left(L_{0,0}\right)$ is $\operatorname{Supp} \sigma\left(L_{0,0}\right)=\left\{\alpha \in \Gamma \mid \sigma\left(L_{0,0}\right)_{\alpha} \neq 0\right\}$. Choose a total order of $\Gamma$ compatible with its group structure such that the maximal element $\alpha_{0}=\max \operatorname{Supp} \sigma\left(L_{0,0}\right)$ of the support of $\sigma\left(L_{0,0}\right)$ is $\succ 0$. Set

$$
\gamma_{0}=\max \operatorname{Supp} \sigma\left(L_{2 \alpha_{0}, 0}\right) .
$$

Since $2 \alpha_{0} \in \operatorname{Supp} \sigma\left(L_{2 \alpha_{0}, 0}\right)$ by (4.3), we have $\gamma_{0} \succeq 2 \alpha_{0} \succ \alpha_{0} \succ 0$. Thus

$$
\left[\sigma\left(L_{2 \alpha_{0}, 0}\right), \sigma\left(L_{0,0}\right)\right]_{\gamma_{0}+\alpha_{0}} \neq 0,
$$

by considering its first term (recall this notion in the proof of Claim 1 in the proof of Theorem 2.1). This shows that $\sigma\left(L_{2 \alpha_{0}, 0}\right)_{\gamma_{0}+\alpha_{0}} \neq 0$, a contradiction with the definition of $\gamma_{0}$ in (4.4). Thus $\sigma\left(L_{0,0}\right)=\sigma\left(L_{0,0}\right)_{0}$ is a homogeneous element concentrated on degree zero. Applying $\sigma$ to $\left[L_{0,0}, L_{\alpha, 0}\right]=\alpha L_{\alpha, 0}$, we see $\sigma\left(L_{\alpha, 0}\right)=\sigma\left(L_{\alpha, 0}\right)_{\alpha}$ is a homogeneous element concentrated on degree $\alpha$ for all $\alpha \in \Gamma$. If $\sigma\left(L_{0,0}\right) \neq L_{0,0}$, by considering the last term of $\left[\sigma\left(L_{0,0}\right), \sigma\left(L_{\alpha, 0}\right)\right]$ with $\alpha \neq 0$, we see $\left[\sigma\left(L_{0,0}\right), \sigma\left(L_{\alpha, 0}\right)\right] \neq \alpha \sigma\left(L_{\alpha, 0}\right)$, a contradiction. The claim is proved.

Applying $\sigma$ to $\left[L_{0,0}, L_{\alpha, 0}\right]=\alpha L_{\alpha, 0}$, by (4.3) and Claim 1, we obtain

$$
\sigma\left(L_{\alpha, 0}\right)=L_{\alpha, 0} \text { for all } \alpha \in \Gamma \text {. }
$$

Now for any $\alpha \in \Gamma$, we set

$$
y^{\alpha}=\sigma\left(L_{\alpha, 1}\right)=\sum_{\beta} y_{\beta}^{\alpha}, \text { where } y_{\beta}^{\alpha}=\sum_{j \geq 0} c_{\beta, j}^{\alpha} L_{\beta, j},
$$

for some $c_{\beta, j}^{\alpha} \in \mathbb{C}$ with $c_{\beta, 0}^{\alpha}=0$ (recall that $\left.\sigma\left(W^{1}\right)=W^{1}\right)$.

Claim 2. We have $y_{\beta}^{\alpha}=0$ if $\beta \neq \alpha$, and $y^{\alpha}=y_{\alpha}^{\alpha}=\sum_{j \geq 1} c_{j} L_{\alpha, j}$ for some $c_{j} \in \mathbb{C}$ with $c_{1} \neq 0$.

Assume $y^{\alpha_{0}}$ is not concentrated on its $\alpha_{0}$-component, i.e., $y^{\alpha_{0}} \neq y_{\alpha_{0}}^{\alpha_{0}}$ for some $\alpha_{0} \in \Gamma$. Let $\beta_{0}$ be the element in $\Gamma^{*}$ (we choose an order of $\Gamma$ such that $\beta_{0} \succ 0$ ) such that

$$
\beta_{0} \succeq \max \operatorname{Supp} y^{0}, \quad \text { or } \beta_{0}+\alpha_{0} \succeq \max \operatorname{Supp} y^{\alpha_{0}}
$$

and at least one of them with equality. Note that

$$
2\left(\alpha_{0}-\gamma\right) L_{\beta, 1}=\left[L_{\beta-\alpha_{0}, 0}, L_{\alpha_{0}, 1}\right]-\left[L_{\beta-\gamma, 0}, L_{\gamma, 1}\right] \text { for } \beta, \gamma \in \Gamma .
$$

First taking $\gamma=0$ and applying $\sigma$ to it, by (4.5) and (4.7), we obtain $y_{\mu}^{\beta}=0$ if $\mu \succ \beta_{0}+\beta$, i.e.,

$$
\max \operatorname{Supp} y^{\beta} \preceq \beta_{0}+\beta \text { for all } \beta \in \Gamma \text {, }
$$


and by computing the coefficient of $L_{\beta_{0}+\beta, j}$, we have (here and below, we simply denote $c_{j}^{\beta}=c_{\beta_{0}+\beta, j}^{\beta}$ for $\left.\beta \in \Gamma\right)$,

$$
c_{j}^{\beta}=\frac{1}{2 \alpha_{0}}\left(\left(2 \alpha_{0}+\beta_{0}-\beta\right) c_{j}^{\alpha_{0}}+(j-1) c_{j-1}^{\alpha_{0}}-\left(\beta_{0}-\beta\right) c_{j}^{0}-(j-1) c_{j-1}^{0}\right)=c_{j}+c_{j}^{\prime} \beta,
$$

where $c_{j}^{\prime}=\frac{1}{2 \alpha_{0}}\left(c_{j}^{0}-c_{j}^{\alpha_{0}}\right), c_{j}=\frac{1}{2 \alpha_{0}}\left(\left(2 \alpha_{0}+\beta_{0}\right) c_{j}^{\alpha_{0}}+(j-1) c_{j-1}^{\alpha_{0}}-\beta_{0} c_{j}^{0}-(j-1) c_{j-1}^{0}\right)$ are some complex numbers not depending on $\beta$ but depending on $j$. Applying $\sigma$ to (4.8) with arbitrary $\gamma$ and equating coefficients of $L_{\beta_{0}+\beta, j}$ gives

$$
2\left(\alpha_{0}-\gamma\right) c_{j}^{\beta}=\left(2 \alpha_{0}+\beta_{0}-\beta\right) c_{j}^{\alpha_{0}}+(j-1) c_{j-1}^{\alpha_{0}}-\left(2 \gamma+\beta_{0}-\beta\right) c_{j}^{\gamma}-(j-1) c_{j-1}^{\gamma} .
$$

Using (4.10) to replace $c_{j}^{\beta}, c_{j}^{\gamma}$ and $c_{j-1}^{\gamma}$ in (4.11), and comparing the coefficients of $\gamma^{2}$, we immediately obtain $c_{j}^{\prime}=0$. Thus $c_{j}^{\beta}=c_{j}$ for all $\beta \in \Gamma$, and from (4.6), we can write

$$
y_{\beta+\beta_{0}}^{\beta}=\sigma\left(L_{\beta, 1}\right)_{\beta+\beta_{0}}=\sum_{j \geq 1} c_{j} L_{\beta+\beta_{0}, j} .
$$

Now apply $\sigma$ to

$$
L_{\alpha, 2}=\left[L_{0,0}, L_{\alpha, 1}\right]-\alpha L_{\alpha, 1}
$$

we see

$$
\max \operatorname{Supp} \sigma\left(L_{\alpha, 2}\right) \preceq \alpha+\beta_{0} \text { for } \alpha \in \Gamma \text {. }
$$

Applying $\sigma$ to

$$
L_{\alpha, 2}=\frac{1}{\alpha-2 \beta}\left[L_{\beta, 1}, L_{\alpha-\beta, 1}\right] \text { with } \alpha \neq 2 \beta,
$$

and computing the first term in its $\left(\alpha+2 \beta_{0}\right)$-th homogeneous component, using (4.12), we see $\alpha+2 \beta_{0} \in \operatorname{Supp} \sigma\left(L_{\alpha, 2}\right)$, a contradiction with (4.14) and the fact that $\beta_{0} \succ 0$. This proves Claim 2 (note that $c_{1} \neq 0$ since $\sigma\left(W^{1}\right)=W^{1} \not \subset W^{2}$ ).

Now from (4.13), we obtain $\sigma\left(L_{\alpha, 2}\right)=\sum_{j \geq 1} j c_{j} L_{\alpha, j+1}$. Using this and (4.9), (4.12) (which is now equal to $y^{\beta}$ ), and applying $\sigma$ to (4.15), we immediately obtain $c_{1}=1$, and $(k-1) c_{k-1}=\sum_{i+j=k} c_{i} c_{j}$ for $k \geq 2$. But the latter relations force $c_{k}=c_{2}^{k-1}$ for all $k \geq 2$. So if $c_{2} \neq 0$, then $\sigma\left(L_{\alpha, 1}\right) \notin W$, a contradiction. Thus $c_{2}=0$ and $\sigma\left(L_{\alpha, 1}\right)=L_{\alpha, 1}$. Since $W$ is generated by $\left\{L_{\alpha, 0}, L_{\alpha, 1} \mid \alpha \in \Gamma\right\}$, we obtain $\sigma=1$. This proves the theorem.

\section{$5 \quad$ Second cohomology group}

Recall that a bilinear form $\psi: W \times W \rightarrow \mathbb{C}$ is called a 2-cocycle on $W$ if the following conditions are satisfied:

$$
\psi(x, y)=-\psi(y, x), \quad \psi(x,[y, z])+\psi(y,[z, x])+\psi(z,[x, y])=0
$$


for $x, y, z \in W$. Denote by $C^{2}(W, \mathbb{C})$ the vector space of 2-cocycles on $W$. For any linear function $f: W \rightarrow \mathbb{C}$, one can define a 2-cocycles $\psi_{f}$ by $\psi_{f}(x, y)=f([x, y])$ for $x, y \in W$. Such a 2-cocycle is called a trivial 2-cocycle or a 2-coboundary on $W$. Denote by $B^{2}(W, \mathbb{C})$ the vector space of 2-coboundaries on $W$. The quotient space

$$
H^{2}(W, \mathbb{C})=C^{2}(W, \mathbb{C}) / B^{2}(W, \mathbb{C})
$$

is called the 2-cohomology group of $W$. There exists a one-to-one correspondence between the set of equivalence classes of one-dimensional central extensions of $W$ by $\mathbb{C}$ and the 2-cohomology group of $W$.

Theorem 5.1. We have $H^{2}(W, \mathbb{C})=\mathbb{C} \bar{\phi}_{0}$, where $\bar{\phi}_{0}$ is the equivalence class of the 2-cocycle $\phi_{0}$, which is defined by

$$
\phi_{0}\left(L_{\alpha, i}, L_{\beta, j}\right)=\delta_{\alpha+\beta, 0} \delta_{i+j, 0} \frac{\alpha^{3}-\alpha}{12} .
$$

Thus $W$ has the unique universal central extension defined by (1.2).

Proof. First note from (4.2) that if $\Gamma^{\prime}=c \Gamma$ for some $c \in \mathbb{C}^{*}$, then $W(\Gamma) \cong W\left(\Gamma^{\prime}\right)$. Thus without loss of generality, we can always suppose $1 \in \Gamma$. Let $\psi \in C^{2}(W, \mathbb{C})$, we define a linear function $f: W \rightarrow \mathbb{C}$ such that $f\left(L_{\alpha, i}\right)$ is defined by induction on $i$ as follows

$$
f\left(L_{\alpha, i}\right)= \begin{cases}\frac{1}{2} \psi\left(L_{-1,0}, L_{1,0}\right) & \text { if } \alpha=i=0, \\ \frac{1}{\alpha} \psi\left(L_{0,0}, L_{\alpha, 0}\right) & \text { if } \alpha \neq 0, i=0, \\ \frac{1}{2}\left(\psi\left(L_{0,0}, L_{0,1}\right)+\psi\left(L_{-1,1}, L_{1,0}\right)\right) & \text { if } \alpha=0, i=1, \\ \frac{1}{2 \alpha}\left(\psi\left(L_{0,0}, L_{\alpha, 1}\right)+\psi\left(L_{0,1}, L_{\alpha, 0}\right)\right) & \text { if } \alpha \neq 0, i=1, \\ \frac{1}{2}\left(\psi\left(L_{0,0}, L_{\alpha, 1}\right)-\psi\left(L_{0,1}, L_{\alpha, 0}\right)\right) & \text { if } i=2, \\ \frac{1}{i-1}\left(\psi\left(L_{0,0}, L_{\alpha, i-1}\right)-\alpha f\left(L_{\alpha, i-1}\right)\right) & \text { if } i \geq 3 .\end{cases}
$$

Set $\phi=\psi-\psi_{f}$. From the second and last cases of (5.2), we obtain

$$
\phi\left(L_{0,0}, L_{\alpha, i}\right)=\psi\left(L_{0,0}, L_{\alpha, i}\right)-f\left(\left[L_{0,0}, L_{\alpha, i}\right]\right)=0 \text { for } \alpha \in \Gamma, 0 \neq i \in \mathbb{Z}_{+} .
$$

Similarly, from the second, fourth and fifth cases of (5.2), we obtain

$$
\begin{aligned}
& \phi\left(L_{0,0}, L_{\alpha, 1}\right)=\psi\left(L_{0,0}, L_{\alpha, 1}\right)-f\left(\left[L_{0,0}, L_{\alpha, 1}\right]\right)=0, \\
& \phi\left(L_{0,1}, L_{\alpha, 0}\right)=\psi\left(L_{0,1}, L_{\alpha, 0}\right)-f\left(\left[L_{0,1}, L_{\alpha, 0}\right]\right)=0 \text { for } \alpha \in \Gamma .
\end{aligned}
$$

From the second and third cases of (5.2),

$$
\phi\left(L_{1,0}, L_{-1,1}\right)=\psi\left(L_{1,0}, L_{-1,1}\right)-f\left(\left[L_{1,0}, L_{-1,1}\right]\right)=0 .
$$


Furthermore by replacing $\phi$ by $\phi-c \phi_{0}$ for some $c \in \mathbb{C}$, we can suppose

$$
\phi\left(L_{\alpha, 0}, L_{\beta, 0}\right)=0 \text { for } \alpha, \beta \in \Gamma .
$$

Then (5.3) gives

$$
0=\phi\left(L_{0,0},\left[L_{\alpha, 0}, L_{\beta, i-1}\right]\right)=(\alpha+\beta) \phi\left(L_{\alpha, 0}, L_{\beta, i-1}\right)+(i-1) \phi\left(L_{\alpha, 0}, L_{\beta, i}\right) .
$$

Now we have

$$
\begin{aligned}
(\alpha-2 \beta) \phi\left(L_{\alpha, 0}, L_{\gamma, 1}\right)= & \phi\left(\left[L_{\beta, 0}, L_{\alpha-\beta, 0}\right], L_{\gamma, 1}\right) \\
= & (\beta-\gamma) \phi\left(L_{\alpha-\beta, 0}, L_{\beta+\gamma, 1}\right)-\phi\left(L_{\alpha-\beta, 0}, L_{\beta+\gamma, 2}\right) \\
& +(\gamma+\beta-\alpha) \phi\left(L_{\beta, 0}, L_{\alpha-\beta+\gamma, 1}\right)+\phi\left(L_{\beta, 0}, L_{\alpha-\beta+\gamma, 2}\right) \\
= & (\alpha+\beta) \phi\left(L_{\alpha-\beta, 0}, L_{\beta+\gamma, 1}\right)+(\beta-2 \alpha) \phi\left(L_{\beta, 0}, L_{\alpha-\beta+\gamma, 1}\right),
\end{aligned}
$$

where the last equality follows from (5.8). Setting $\beta=-\alpha$ in (5.9) gives

$$
\phi\left(L_{-\alpha, 0}, L_{\gamma+2 \alpha, 1}\right)=-\phi\left(L_{\alpha, 0}, L_{\gamma, 1}\right) .
$$

Setting $\beta=-\gamma$ in (5.9), by (5.5) and (5.10), we obtain

$$
(\alpha+2 \gamma) \phi\left(L_{\alpha, 0}, L_{\gamma, 1}\right)=(\gamma+2 \alpha) \phi\left(L_{\gamma, 0}, L_{\alpha, 1}\right) .
$$

Exchanging $\alpha$ and $\gamma$ and changing $\beta$ to $-\beta$ in (5.9), by (5.10) and (5.11), we obtain

$$
\begin{aligned}
& (\gamma+2 \beta) \frac{\alpha+2 \gamma}{\gamma+2 \alpha} \phi\left(L_{\alpha, 0}, L_{\gamma, 1}\right) \\
& =(\gamma-\beta) \phi\left(L_{\gamma+\beta, 0}, L_{\alpha-\beta, 1}\right)+(\beta+2 \gamma) \phi\left(L_{\beta, 0}, L_{\gamma+\alpha-\beta, 1}\right) \\
& =(\gamma-\beta) \frac{\alpha+\beta+2 \gamma}{2 \alpha-\beta+\gamma} \phi\left(L_{\alpha-\beta, 0}, L_{\gamma+\beta, 1}\right)+(\beta+2 \gamma) \phi\left(L_{\beta, 0}, L_{\gamma+\alpha-\beta, 1}\right),
\end{aligned}
$$

for all $\alpha, \beta, \gamma \in \Gamma$ (note that if $\alpha=-2 \gamma$ or $\beta=2 \alpha+\gamma$, we shall regard the equation as the one by first multiplying the equation by $\alpha+2 \gamma$ or $2 \alpha-\beta+\gamma$, then take $\alpha=-2 \gamma$ or $\beta=2 \alpha+\gamma)$. Taking $\beta=1$, we can solve $\phi\left(L_{\alpha, 0}, L_{\gamma, 1}\right)$ from (5.9) and (5.12) to obtain

$$
\phi\left(L_{\alpha, 0}, L_{\gamma, 1}\right)=\frac{\alpha \gamma(2 \alpha+\gamma)}{(\alpha+\gamma+1)(\alpha+\gamma-1)} c_{\alpha+\gamma} \text { if } \alpha \neq-\gamma \pm 1,
$$

where $c_{\alpha}=\phi\left(L_{1,0}, L_{\alpha-1,1}\right) \in \mathbb{C}$. In particular, by (5.6),

$$
\phi\left(L_{\alpha, 0}, L_{\gamma, 1}\right)=0 \quad \text { if } \quad \alpha+\gamma=0 .
$$

Now (5.3) gives

$$
0=\phi\left(L_{0,0},\left[L_{\alpha, 1}, L_{\beta, 1}\right]\right)=(\alpha+\beta) \phi\left(L_{\alpha, 1}, L_{\beta, 1}\right)+\phi\left(L_{\alpha, 2}, L_{\beta, 1}\right)+\phi\left(L_{\alpha, 1}, L_{\beta, 2}\right) .
$$


Equation (5.8) gives

$$
\begin{aligned}
& -(\alpha+\beta) \beta \phi\left(L_{\alpha, 0}, L_{\beta, 1}\right) \\
& =\beta \phi\left(L_{\alpha, 0}, L_{\beta, 2}\right)=\phi\left(L_{\alpha, 0},\left[L_{0,1}, L_{\beta, 1}\right]\right) \\
& =-\alpha \phi\left(L_{\alpha, 1}, L_{\beta, 1}\right)+\phi\left(L_{\alpha, 2}, L_{\beta, 1}\right)+(\beta-\alpha) \phi\left(L_{0,1}, L_{\alpha+\beta, 1}\right)+\phi\left(L_{0,1}, L_{\alpha+\beta, 2}\right) .
\end{aligned}
$$

Solving $\phi\left(L_{\alpha, 2}, L_{\beta, 1}\right)$ in this equation gives

$$
\begin{aligned}
& \phi\left(L_{\alpha, 2}, L_{\beta, 1}\right) \\
= & \alpha \phi\left(L_{\alpha, 1}, L_{\beta, 1}\right)+(\alpha-\beta) \phi\left(L_{0,1}, L_{\alpha+\beta, 1}\right)-\phi\left(L_{0,1}, L_{\alpha+\beta, 2}\right)-(\alpha+\beta) \beta \phi\left(L_{\alpha, 0}, L_{\beta, 1}\right) .
\end{aligned}
$$

Thus we also have an expression for $\phi\left(L_{\alpha, 1}, L_{\beta, 2}\right)=-\phi\left(L_{\beta, 2}, L_{\alpha, 1}\right)$. Using them in (5.15), by (5.11), we obtain

$$
\phi\left(L_{\alpha, 1}, L_{\beta, 1}\right)=\frac{\beta^{2}-\alpha^{2}}{2(2 \alpha+\beta)} \phi\left(L_{\alpha, 0}, L_{\beta, 1}\right)+\frac{\beta-\alpha}{\alpha+\beta} \phi\left(L_{0,1}, L_{\alpha+\beta, 1}\right) \text { if } \alpha \neq-\beta \text {. }
$$

Using (5.8), we have

$$
\begin{aligned}
& (\beta-\gamma)(\alpha+\beta+\gamma) \phi\left(L_{\alpha, 0}, L_{\beta+\gamma, 1}\right) \\
& =(\gamma-\beta) \phi\left(L_{\alpha, 0}, L_{\beta+\gamma, 2}\right) \\
& =\phi\left(L_{\alpha, 0},\left[L_{\beta, 1}, L_{\gamma, 1}\right]\right) \\
& =(\beta-\alpha) \phi\left(L_{\alpha+\beta, 1}, L_{\gamma, 1}\right)+\phi\left(L_{\alpha+\beta, 2}, L_{\gamma, 1}\right)+(\gamma-\alpha) \phi\left(L_{\beta, 1}, L_{\alpha+\gamma, 1}\right)+\phi\left(L_{\beta, 1}, L_{\alpha+\gamma, 2}\right) .
\end{aligned}
$$

Using (5.13), (5.17) and (5.18) in (5.19), we can solve $c_{\alpha}=0$, thus (5.13), (5.18) and (5.17) give

$$
\begin{aligned}
& \phi\left(L_{\alpha, 0}, L_{\beta, 1}\right)=0, \quad \phi\left(L_{\alpha, 1}, L_{\beta, 1}\right)=\frac{\beta-\alpha}{\alpha+\beta} \tilde{c}_{\alpha+\beta} \quad(\text { if } \alpha \neq-\beta), \\
& \phi\left(L_{\alpha, 1}, L_{\beta, 2}\right)=\frac{\alpha(\alpha-\beta)}{\alpha+\beta} \tilde{c}_{\alpha+\beta}+\bar{c}_{\alpha+\beta} \quad(\text { if } \alpha \neq-\beta),
\end{aligned}
$$

where $\tilde{c}_{\alpha}=\phi\left(L_{0,1}, L_{\alpha, 1}\right), \bar{c}_{\alpha}=\phi\left(L_{0,1}, L_{\alpha, 2}\right) \in \mathbb{C}$ (note that although we obtain the above under some conditions (for instance, there is a condition in (5.13)), by (5.9), all conditions can be removed as long as the expressions make senses). Now using (5.21) in

$(\gamma-\beta) \phi\left(L_{\alpha, 1}, L_{\beta+\gamma, 2}\right)=\phi\left(L_{\alpha, 1},\left[L_{\beta, 1}, L_{\gamma, 1}\right]\right)=(\beta-\alpha) \phi\left(L_{\alpha+\beta, 2}, L_{\gamma, 1}\right)+(\gamma-\alpha) \phi\left(L_{\beta, 1}, L_{\alpha+\gamma, 2}\right)$, we obtain $\tilde{c}_{\alpha}=0$. Thus

$$
\phi\left(L_{\alpha, 1}, L_{\beta, 1}\right)=0, \quad \phi\left(L_{\alpha, 1}, L_{\beta, 2}\right)=\bar{c}_{\alpha+\beta}
$$


Using (5.8) and (5.20), by induction on $i$, we obtain $\phi\left(L_{\alpha, 0}, L_{\beta, i}\right)=0$. Thus

$$
0=\phi\left(L_{\alpha, 0},\left[L_{\beta, 1}, L_{\gamma, 2}\right]\right)=(\beta-\alpha) \bar{c}_{\alpha+\beta+\gamma}+\phi\left(L_{\alpha+\beta, 2}, L_{\gamma, 2}\right)+(\gamma-\alpha) \bar{c}_{\alpha+\beta+\gamma}+2 \phi\left(L_{\beta, 1}, L_{\alpha+\gamma, 3}\right) .
$$

Setting $\alpha=-\beta$ and $\beta=0$ respectively, we can solve

$$
\phi\left(L_{\alpha, 1}, L_{\beta, 3}\right)=-\frac{(4 \alpha+\beta)}{2} \bar{c}_{\alpha+\beta}-\frac{1}{2} \hat{c}_{\alpha+\beta}, \quad \phi\left(L_{\alpha, 2}, L_{\beta, 2}\right)=3 \alpha \bar{c}_{\alpha+\beta}+\hat{c}_{\alpha+\beta},
$$

where $\hat{c}_{\alpha}=\phi\left(L_{0,2}, L_{\alpha, 2}\right)$. Since $\phi\left(L_{\alpha, 2}, L_{\beta, 2}\right)=-\phi\left(L_{\beta, 2}, L_{\alpha, 2}\right)$, we obtain $\hat{c}_{\alpha}=-\frac{3}{2} \alpha \bar{c}_{\alpha}$. Using (5.23) in

$$
\begin{aligned}
(\beta-\alpha) \phi\left(L_{0,1}, L_{\alpha+\beta, 3}\right)+\phi\left(L_{0,1}, L_{\alpha+\beta, 4}\right) & =\phi\left(L_{0,1},\left[L_{\alpha, 1}, L_{\beta, 2}\right]\right) \\
& =\alpha \phi\left(L_{\alpha, 2}, L_{\beta, 2}\right)+\beta \phi\left(L_{\alpha, 1}, L_{\beta, 3}\right)+\phi\left(L_{\alpha, 1}, L_{\beta, 4}\right),
\end{aligned}
$$

we solve

$$
\phi\left(L_{\alpha, 1}, L_{\beta, 4}\right)=\frac{\alpha}{4}(11 \beta-7 \alpha) \bar{c}_{\alpha+\beta}+c_{\alpha+\beta}^{\prime},
$$

where $c_{\alpha}^{\prime}=\phi\left(L_{0,1}, L_{\alpha, 4}\right) \in \mathbb{C}$. Using this and (5.23) in

$$
0=\phi\left(L_{0,0},\left[L_{\alpha, 1}, L_{\beta, 3}\right]\right)=(\alpha+\beta) \phi\left(L_{\alpha, 1}, L_{\beta, 3}\right)+\phi\left(L_{\alpha, 2}, L_{\beta, 3}\right)+3 \phi\left(L_{\alpha, 1}, L_{\beta, 4}\right)
$$

we obtain

$$
\phi\left(L_{\alpha, 2}, L_{\beta, 3}\right)=\frac{1}{4}\left(26 \alpha^{2}-29 \alpha \beta-\beta^{2}\right) \bar{c}_{\alpha+\beta}-3 c_{\alpha+\beta}^{\prime} .
$$

Using this and (5.23) in

$$
0=\phi\left(L_{0,0},\left[L_{\alpha, 2}, L_{\beta, 2}\right]\right)=(\beta+\alpha) \phi\left(L_{\alpha, 2}, L_{\beta, 2}\right)+2 \phi\left(L_{\alpha, 3}, L_{\beta, 2}\right)+2 \phi\left(L_{\alpha, 2}, L_{\beta, 3}\right),
$$

we immediately obtain $\bar{c}_{\alpha}=0$. This proves

$$
\phi\left(L_{\alpha, i}, L_{\beta, j}\right)=0 \quad \text { for } \quad i+j \leq 4
$$

Now inductively assume for $k \geq 4$, we have proved $\phi\left(L_{\alpha, i}, L_{\beta, j}\right)=0$ for all $i+j \leq k$. Then

$$
0=\phi\left(L_{\alpha, 0},\left[L_{\beta, i}, L_{\gamma, k-i}\right]\right)=i \phi\left(L_{\alpha+\beta, i+1}, L_{\gamma, k-i}\right)+(k-i) \phi\left(L_{\beta, i}, L_{\alpha+\gamma, k-i+1}\right) .
$$

Setting $\beta=0$ and $\gamma=0$ in (5.26) respectively, we can solve

$$
\phi\left(L_{\alpha, i}, L_{\beta, k+1-i}\right)=(-1)^{i-1}\left(\begin{array}{c}
k-1 \\
i-1
\end{array}\right) c_{\alpha+\beta}^{k} \text { for some } c_{\alpha}^{k} \in \mathbb{C} .
$$


If $k=2 \ell-1$ is odd, then since $\phi\left(L_{\alpha, \ell}, L_{\beta, \ell}\right)=-\phi\left(L_{\beta, \ell}, L_{\alpha, \ell}\right)$, we obtain $c_{\alpha}^{k}=0$ by $(5.27)$, and thus $\phi\left(L_{\alpha, i}, L_{\beta, k+1-i}\right)=0$. Now assume $k=2 \ell$ is even. Using (5.27), we have

$$
\begin{aligned}
0= & \phi\left(L_{\alpha, 0},\left[L_{\beta, \ell}, L_{\gamma, \ell+1}\right]\right) \\
= & (\beta-\alpha) \phi\left(L_{\alpha+\beta, \ell}, L_{\gamma, \ell+1}\right)+\ell \phi\left(L_{\alpha+\beta, \ell+1}, L_{\gamma, \ell+1}\right) \\
& +(\gamma-\alpha) \phi\left(L_{\beta, \ell}, L_{\alpha+\gamma, \ell+1}\right)+(\ell+1) \phi\left(L_{\beta, \ell}, L_{\alpha+\gamma, \ell+2}\right) \\
= & (\beta+\gamma-2 \alpha) \phi\left(L_{0, \ell}, L_{\alpha+\beta+\gamma, \ell+1}\right) \\
& +\ell \phi\left(L_{\alpha+\beta, \ell+1}, L_{\gamma, \ell+1}\right)+(\ell+1) \phi\left(L_{\beta, \ell}, L_{\alpha+\gamma, \ell+2}\right) .
\end{aligned}
$$

Setting $\beta=0$, we obtain

$$
0=(\gamma-2 \alpha) \phi\left(L_{0, \ell}, L_{\alpha+\gamma, \ell+1}\right)+\ell \phi\left(L_{\alpha, \ell+1}, L_{\gamma, \ell+1}\right)+(\ell+1) \phi\left(L_{0, \ell}, L_{\alpha+\gamma, \ell+2}\right) .
$$

Exchanging $\alpha$ and $\gamma$, and summing the result with (5.29), by the skew symmetry of $\phi\left(L_{\alpha, \ell+1}, L_{\gamma, \ell+1}\right)$, we obtain

$$
\phi\left(L_{0, \ell}, L_{\alpha, \ell+2}\right)=\frac{\alpha}{2(\ell+1)} \phi\left(L_{0, \ell}, L_{\alpha, \ell+1}\right) .
$$

Using this in (5.29) gives $\phi\left(L_{\alpha, \ell+1}, L_{\gamma, \ell+1}\right)=\frac{3(\gamma-\alpha)}{2 \ell} \phi\left(L_{0, \ell}, L_{\alpha+\gamma, \ell+1}\right)$. Using this in (5.28), we obtain

$$
0=\frac{5 \gamma-\beta-7 \alpha}{2} \phi\left(L_{0, \ell}, L_{\alpha+\beta+\gamma, \ell+1}\right)+(\ell+1) \phi\left(L_{\beta, \ell}, L_{\alpha+\gamma, \ell+2}\right)
$$

Exchanging $\alpha$ and $\gamma$ shows $\phi\left(L_{0, \ell}, L_{\alpha+\beta+\gamma, \ell+1}\right)=0$. This together with (5.27) proves $c_{\alpha}^{k}=0$, and thus $\phi\left(L_{\alpha, i}, L_{\beta, k+1-i}\right)=0$. The theorem is proved by induction.

\section{References}

[1] R. Farnsteiner, Derivations and central extensions of finitely generated graded Lie algebras, J. Algebra, 118 (1988), 33-45.

[2] V.G. Kac, Infinite-dimensional Lie algebras, Third edition, Cambridge University Press, Cambridge, 1990.

[3] V.G. Kac, A. Raina, Bombay Lectures on Highest Weight Representations of Infinite Dimensional Lie Algebras, Adv. Ser. Math. Phys., Vol 2. World Sci.

[4] J. Patera and H. Zassenhaus, The higher rank Virasoro algebras, Comm. Math. Phys., 136 (1991), 1-14.

[5] Y. Su, Classification of Harish-Chandra modules over the higher rank Virasoro algebras, Comm. Math. Phys. 240 (2003), 539-551.

[6] Y. Su, Quasifinite representations of a Lie algebra of Block type, J. Algebra 276 (2004), 117-128. 
[7] Y. Su, Quasifinite representations of a family of Lie algebras of Block type, J. Pure Appl. Algebra 192 (2004), 293-305.

[8] Y. Su, X. Xu, H. Zhang, Derivation-simple algebras and the structures of Lie algebras of Witt type, J. Algebra, 233 (2000), 642-662.

[9] Y. Su, K. Zhao, Second cohomology group of generalized Witt type Lie algebras and certain representations, Comm. Algebra, 30 (2002), 3285-3309.

[10] Y. Su, K. Zhao, Generalized Virasoro and super-Virasoro algebras and modules of the intermediate series, J. Algebra, 252 (2002), 1-19.

[11] H. Wu, S. Wang, X. Yue, Structures of generalized loop Virasoro algebras, Comm. Algebra, 42 (2013), 1545-1558.

[12] X. Xu, New generalized simple Lie algebras of Cartan type, J. Algebra, 224 (2000), 23-58.

[13] W. Zhang, C. Dong, W-algebra W(2,2) and the vertex operator algebra $L(1 / 2,0) \otimes L(1 / 2,0)$, Comm. Math. Phys. 285 (2009), 991-1004. 